\title{
Controle de Senecio spp. com pastoreio ovino de acordo com sua infestação e fenologia ${ }^{1}$
}

\author{
Fernando Castilhos Karam²*, José Carlos Ferrugem Moraes $^{3}$ e Ana Lucia Schild ${ }^{4}$
}

\begin{abstract}
Karam F.C., Moraes J.C. F. \& Schild A.L. 2013. [Sheep grazing for control of Senecio spp. according to infestation level and phenology.] Controle de Senecio spp. com pastoreio ovino de acordo com sua infestação e fenologia. Pesquisa Veterinária Brasileira 33(9):1109-1115. Instituto de Pesquisas Veterinárias Desidério Finamor, Fundação Estadual de Pesquisa Agropecuária, Estrada do Conde 6000, Eldorado do Sul, RS 92990-000, Brazil. E-mail: fernandockaram@yahoo.com.br
\end{abstract}

Senecio spp. poisoning is the major cause of death among cattle in Rio Grande do Sul (RS), Brazil. There are no efficient therapeutic measures, but sheep are more resistant and have been used as natural control. The aim of this paper was to assess the efficiency of sheep grazing in different seasons of the year (spring-winter), determining the best time for interference in plant phenology, especially in reproductive phases, and relative Senecio infestation in the pasture. The experiment was conducted on two farms located in the Campanha region of RS, in 50x100m plots, with water availability and with different levels of infestation by Senecio spp. The level of infestation, regardless of the species, was determined by the average number of plants at three random sites measuring $2 \mathrm{~m}$ in diameter each, within the area of study. In 12 phenological observation points defined in each plot, one to three plants showed low infestation (IB) and four to six plants revealed average infestation (IM), both located on farm A; seven to nine plants had high infestation (IA) and 10 or more plants showed very high infestation (IMA), located on farm B. The plots were on different farms because it was not possible to find all infestation levels on a single farm. The following parameters were evaluated on day 0 (before the introduction of sheep) and every 15 days during the spring of 2009 (year 1) and the winter and spring of 2010 (year 2): number of plants of the Senecio species, reproductive phenophases, vigor, and plant consumption by the sheep. Control was carried out in year 2, in six phenological observation points, which were identical and contiguous to each plot, without sheep grazing, with regular cattle and horse grazing, following the rotation scheme used on the farms. At baseline, each control had the same level of infestation by Senecio spp. corresponding to the experimental area. Between the spring of 2009 and the winter of 2010, the sheep were removed and both cattle and horses were allowed to graze on the plots. The statistical analysis consisted of simple regression and the means were compared by covariance. Reproductive phenophases, vigor, and plant consumption by the sheep, which did not have a normal distribution, were compared by Wilcoxon nonparametric test. The results demonstrated that sheep grazing had a negative impact on the development of Senecio species and that, in winter, it inhibits their future reproductive phenophases. Given the phenological and environmental conditions, as well as the risk for the cattle, the control of Senecio spp. by sheep grazing was more efficient in winter than in spring. Due to the negative impact on plant growth,

\footnotetext{
${ }^{1}$ Recebido em 17 de julho de 2013.

Aceito para publicação em 24 de agosto de 2013.

Parte da Tese de Doutorado do primeiro autor, Programa de Pós-Graduação em Veterinária, Faculdade de Veterinária, Universidade Federal de Pelotas (UFPel), Campus Universitário s/n, Pelotas, RS 96010-900, Brasil.

${ }^{2}$ Instituto de Pesquisas Veterinárias Desidério Finamor (IPVDF), Fepa-
}

\footnotetext{
gro, Estrada do Conde 6000, Eldorado do Sul, RS 92990-000, Brasil. *Autor para correspondência: fernandockaram@yahoo.com.br

${ }^{3}$ Embrapa Pecuária Sul, Cx. Postal 242, Bagé, RS 96401-970, Brasil.

${ }^{4}$ Laboratório Regional de Diagnóstico, Faculdade de Veterinária, Universidade Federal de Pelotas (UFPel), Campus Universitário s/n, Pelotas, RS 96010-900.
} 
mainly with regard to reproductive phenophases, with reduction of seed production, sheep grazing is an alternative for the biological control of Senecio spp., which will contribute to reduce their infestation in the medium and long term in RS and, consequently, the damage to cattle breeding.

INDEX TERMS: Seneciosis, Senecio spp., environmental, control measures, sheep.

RESUMO.- Intoxicação por Senecio spp. é a principal causa de morte de bovinos no Rio Grande do Sul (RS) e não há medidas terapêuticas eficazes, mas os ovinos são mais resistentes e têm sido usados como controladores naturais da planta. 0 objetivo desse trabalho foi avaliar a eficiência do pastoreio com ovinos em diferentes estações do ano (primavera-inverno) determinando a melhor época pela interferência desse pastoreio na fenologia da planta, especialmente nas fases reprodutivas, e em relação à infestação na pastagem. 0 experimento foi desenvolvido em duas propriedades localizadas na região da Campanha do RS, em módulos de 50m X 100m, com água disponível e com diferentes graus de infestação por Senecio spp. 0 grau de infestação, independentemente da espécie, foi determinado pela média do número de plantas em três pontos aleatórios com diâmetro de $2 \mathrm{~m}$ cada, dentro da área de estudo. Em 12 pontos de observação fenológica definidos em cada módulo, uma a três plantas representou infestação baixa (IB) e quatro a seis plantas infestação média (IM), ambos localizados na propriedade A; sete a nove plantas infestação alta (IA) e 10 ou mais plantas foi considerada infestação muito alta (IMA), localizados na propriedade B. Os módulos localizaram-se em propriedades distintas porque não foram encontrados todos os níveis de infestação numa única propriedade. Nesses pontos foram avaliados os seguintes parâmetros no dia 0 (antes da entrada dos ovinos) e a cada 15 dias durante a primavera de 2009 (primeiro ano) e inverno e primavera de 2010 (segundo ano): número de exemplares das espécies de Senecio presentes, fenofases reprodutivas, vigor e consumo. 0 controle foi feito no segundo ano do experimento, em seis pontos de observação fenológica, estabelecidos de forma idêntica ao módulo, em área contígua a cada um dos módulos, sem pastoreio ovino, com pastoreio periódico de bovinos e equinos, seguindo o manejo de rodízio aplicado nas propriedades. No momento da instalação, cada controle tinha o mesmo grau de infestação de Senecio spp. correspondente à área experimental. No intervalo de tempo experimental, entre a primavera de 2009 e o inverno de 2010, os ovinos foram retirados e os módulos abertos ao pastoreio de bovinos e equinos. A análise estatística foi feita através de regressão simples e a comparação das médias por covariância, sendo que as variáveis fenofase reprodutiva, vigor e consumo, que não apresentaram distribuição normal, foram comparadas pelo teste não paramétrico de Wilcoxon. Os resultados demonstraram que o pastoreio ovino interferiu negativamente no desenvolvimento das espécies de Senecio acompanhadas e que, no inverno, inibe as futuras fenofases reprodutivas das plantas. Considerando as condições fenológicas, ambientais e de maior risco para os bovinos, o controle de Senecio spp. com ovinos, no inverno, é mais eficaz do que na primavera. Pela interferência negativa na planta, especialmente em relação às fenofases reprodutivas, diminuindo a pro- dução de sementes, o pastoreio ovino é uma alternativa de controle biológico de Senecio spp. que reduzirá a infestação a médio e longo prazo no RS, consequentemente, o prejuízo à bovinocultura.

TERMOS DE INDEXAÇÃO: Seneciose, Senecio spp., ambiente, medidas de controle, ovinos.

\section{INTRODUÇÃO}

Seneciose é a mais frequente das intoxicações por plantas e a principal causa de morte de bovinos no Rio Grande do Sul (RS) (Karam \& Motta 2011). Diversos estudos a respeito dessa toxicose têm sido realizados (Basile et al. 2005, Pedroso et al. 2007, Rissi et al. 2007, Santos et al. 2008, Grecco et al. 2010, Lucena et al. 2010a,b, Karam et al. 2011b), porém a ineficácia de medidas terapêuticas para a intoxicação justifica a busca de alternativas para o controle de Senecio spp. Entre alguns estudos está o da fenologia que possibilitou conhecer o ciclo biológico de quatro espécies do gênero, S. brasiliensis, S. selloi, S. oxyphyllus e S. heterotrichius no RS e forneceu dados importantes para se estabelecer estratégias para o controle através de medidas agronômicas ou biológicas (Karam et al. 2002). Como controle biológico inclui-se o pastoreio ovino (Méndez \& Riet-Correa 2008). Embora os ovinos possam adoecer espontaneamente (Ilha et al. 2001, Grecco et al. 2011), a intoxicação não é comum nessa espécie por ser mais resistente à ação dos diversos alcaloides tóxicos da planta. No RS, nas áreas onde há ovinos em pastoreio, geralmente não há Senecio spp. (Méndez \& Riet-Correa 2008).

Estudos recentes reforçam a ideia de que a expansão de algumas espécies de Senecio está ligada a fatores ambientais como sobrepastoreio e cobertura do solo (Boldrini et al. 2010). A espécie $S$. madagascariensis sofreu um processo de naturalização (Matzenbacher et al. 2011) e está em franca expansão no RS (Matzenbacher 1998, Matzenbacher \& Schneider 2008). É responsável por surto de intoxicação (Cruz et al. 2010) e objeto de estudo sobre dinâmica populacional que sugere a espécie como uma ameaça às plantas nativas e ao gado no RS (Mäder et al. 2011).

O objetivo do presente trabalho foi testar o efeito do pastoreio com ovinos para o controle de Senecio spp. em diferentes estações do ano (primavera-inverno) determinando a melhor época pela interferência na fenologia da planta, especialmente na fase reprodutiva, e em relação ao grau de infestação na pastagem.

\section{MATERIAL E MÉTODOS}

0 estudo foi feito em dois experimentos (1 e 2) desenvolvidos em duas propriedades (A e B) localizadas na região da Campanha do RS, nos município de Aceguá (Propriedade A: latitude 31²44 651', longitude $54^{\circ} 08$ 844' e altitude de $169 \mathrm{~m}$ ) e Bagé (Propriedade B: latitude $31^{\circ} 24703^{\prime}$, longitude $54^{\circ} 06315^{\prime}$ e altitude de $212 \mathrm{~m}$ ), 
em módulos de 50x100m, com água disponível e com diferentes graus de infestação por Senecio spp. Em cada módulo foram colocados dois ovinos da raça Corriedale, machos, castrados, de aproximadamente dois anos de idade. Para definir o grau de infestação por Senecio, independentemente da espécie, foi feita a média do número de plantas em três pontos aleatórios com diâmetro de $2 \mathrm{~m}$ cada, dentro da área de estudo. Em 12 pontos $(2 \mathrm{~m}$ de diâmetro cada) de observação fenológica definidos em cada módulo, uma a três plantas representou infestação baixa (IB) e quatro a seis plantas infestação média (IM), ambos localizados na propriedade A; sete a nove plantas infestação alta (IA) e 10 ou mais plantas foi considerada infestação muito alta (IMA), localizados na propriedade B. Os módulos localizaram-se em propriedades distintas porque não foram encontrados todos os níveis de infestação numa única propriedade. Os pontos de observação fenológica foram assim estabelecidos: em um dos lados de $100 \mathrm{~m}$, a cada $25 \mathrm{~m}, \mathrm{o}$ módulo foi marcado originando três linhas centrais. Perpendicularmente a cada linha, a cada $10 \mathrm{~m}$, foram marcados quatro pontos resultando nos 12 pontos em cada módulo. Nesses pontos foram avaliados os seguintes parâmetros no dia 0 (antes da entrada dos ovinos) e a cada 15 dias durante a primavera de 2009 (Experimento 1) e inverno e primavera de 2010 (Experimento 2): número de exemplares das espécies de Senecio presentes ( $S$. brasiliensis, $S$. madagascariensis e $S$. oxyphyllus), fenofases reprodutivas (não ocorrência=0; botões florais, flores e frutos verdes $=1$; frutos maduros e dispersão de sementes=2), vigor (fraco=1, modera$\mathrm{do}=2$ e forte $=3$ ) e consumo (não ocorrência $=0$, apenas ponteiros das plantas $=1$, parcial de ponteiros e partes aéreas $=2$, total das partes aéreas=3) dos exemplares de Senecio spp. 0 controle foi feito no Experimento 2, em seis pontos de observação fenológica, estabelecidos de forma idêntica ao módulo, em área contígua a cada um dos módulos, sem pastoreio ovino, com pastoreio periódico de bovinos e equinos, seguindo o manejo de rodízio aplicado nas propriedades. No momento da instalação, cada controle tinha o mesmo grau de infestação de Senecio spp. correspondente à área experimental. Cabe salientar que entre os 105 dias de avaliação do Experimento 2, o período compreendido entre 0-60 foi o inverno e o de 75-105 o de primavera. No intervalo de tempo experimental, entre a primavera de 2009 e o inverno de 2010, os ovinos foram retirados e os módulos abertos ao pastoreio de bovinos e equinos. Durante os dois anos experimentais foram obtidos os registros climáticos de temperatura e de precipitação, para a região, pelo Centro Estadual de Meteorologia (CEMETRS/Fepagro. Porto Alegre, RS, Brasil) e a precipitação, também, através de pluviômetro em cada propriedade.

A análise estatística foi feita através de regressão simples e a comparação das médias por covariância, usando o teste não paramétrico de Wilcoxon para as variáveis qualitativas fenofase reprodutiva, vigor e consumo, que não apresentaram distribuição normal. As análises foram procedidas segundo as recomendações de Callegari-Jacques (2003), e efetuadas no R (2011).

\section{RESULTADOS}

Nos dois anos do estudo houve um período seco de março a dezembro de 2010 e a precipitação abaixo da normal em praticamente todo esse período, com exceção do mês de julho. A temperatura ficou ligeiramente acima da normal, na maior parte desse período. Não foi detectada associação da precipitação com o número de plantas e o pastejo de ovinos no período estudado.

No Experimento 1 houve redução do número de plantas ao longo do observado (primavera de 2009), nos quatro módulos das diferentes infestações submetidos ao pasto-

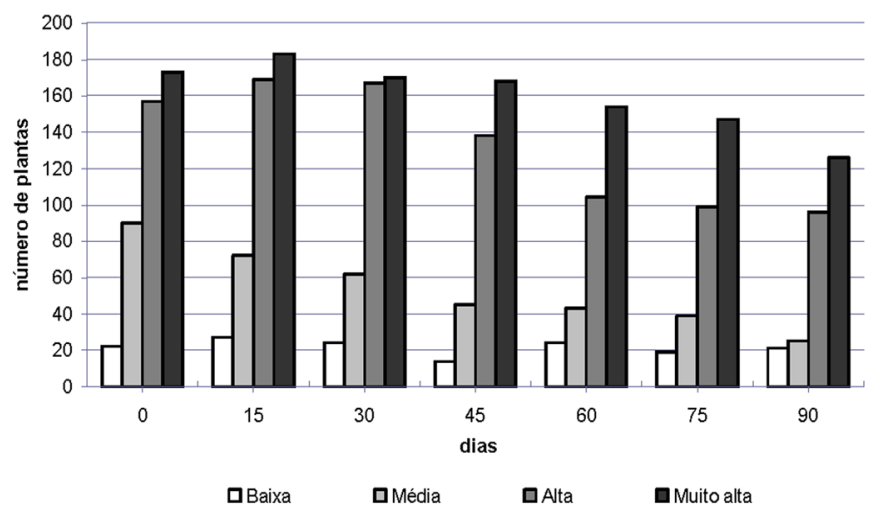

Fig.1. Variação no número de plantas de Senecio spp. em módulos com diferentes níveis de Infestação (Baixa, Média, Alta e Muito alta) submetidos ao pastoreio ovino durante a primavera de 2009 (Experimento 1).

reio ovino (Fig.1). 0 número médio de plantas nos 90 dias de avaliação (13 set. 2009 a 12 dez. 2009), nos quatro níveis de infestação arbitrados Baixa, Média, Alta e Muito Alta foi respectivamente de $21,6 \pm 4,2,53,7 \pm 22,2,132,9 \pm 32,7$ e $160,1 \pm 19,3$. No Quadro 1 são apresentadas as regressões lineares que expressam a redução significativa no número de plantas nos intervalos de leituras quinzenais para as infestações Média, Alta e Muito Alta sob efeito de pastejo ovino. Apenas na infestação Baixa não foi detectada uma redução significativa no número de plantas. A análise de covariância combinada indicou que as médias do número de plantas são diferentes $(\mathrm{F}=18,48903 ; P<0,001)$, mas com inclinações semelhantes $(\mathrm{F}=1,17931 ; P>0,05)$.

Quadro 1. Associação através de regressão simples do número de plantas de Senecio spp. sobre o dia de avaliação, nos diferentes níveis de infestação (Baixa, Média, Alta e Muito alta) submetidos ao pastoreio ovino durante a primavera de 2009 (Experimento 1)

\begin{tabular}{lccc}
\hline Nível de infestação & Intercepto & Inclinação & $\begin{array}{c}\text { Coeficiente de } \\
\text { variação (R2) }\end{array}$ \\
\hline Baixa & $23,60^{* * *}$ & $-0,045 \mathrm{NS}$ & 0,12 \\
Média & $83,714^{* * *}$ & $-0,667^{* * *}$ & 0,94 \\
Alta & $174,21^{* * *}$ & $-0,919^{* *}$ & 0,83 \\
Muito Alta & $184,68^{* * *}$ & $-0,545^{* *}$ & 0,84
\end{tabular}

$\overline{\mathrm{NS}}=$ Não significativo; ${ }^{* *} \mathrm{P}<0,05 ;{ }^{* * *} \mathrm{P}<0,001$.

No Experimento 2 foi observada redução pelo pastejo do número médio de plantas no período avaliado (inverno-primavera de 2010), nos quatro módulos com diferentes infestações (Quadro 2). Na Figura 2 é apresentada a relação entre o número de plantas ao longo dos 105 dias de avaliação, nos intervalos de leituras quinzenais (6 jul. 2010 a 19 out. 2010), nos módulos de pastejo e controle. Os resultados mostram consumo significativo em todos os níveis de infestação. Como peculiaridade a inclinação no tratamento sob pastejo não é significativa no nível de infestação Muito alta (Quadro 2).

As distribuições das variáveis qualitativas fenofases reprodutivas, vigor e consumo foram comparadas nos diferentes níveis de infestação, considerando as observações nos módulos sob pastejo e respectivos controles (Quadro 
Quadro 2. Médias e a associação através de regressão linear do número de plantas de Senecio spp. sobre o dia de avaliação, nos diferentes níveis de infestação (Baixa, Média, Alta e Muito alta) submetidos ao pastoreio ovino e respectivos controles, durante o inverno e primavera de 2010 (Experimento 2)

\begin{tabular}{cccccc}
\hline $\begin{array}{c}\text { Nível de } \\
\text { infestação }\end{array}$ & Tratamento & $\begin{array}{c}\text { Número } \\
\text { médio } \\
\text { de plantas }\end{array}$ & Intercepto & Inclinação & $\begin{array}{c}\text { Coefici- } \\
\text { ente vari- } \\
\text { ação (R2) }\end{array}$ \\
\hline Baixa & Controle & $55,5 \pm 19,6$ & $28,33^{* * *}$ & $0,508^{* * *}$ & 0,90 \\
& Pastejo & $19,1 \pm 9,5$ & $30,50^{* * *}$ & $-0,217^{* *}$ & 0,71 \\
Média & Controle & $76,5 \pm 13,7$ & $60,50^{* * *}$ & $0,305^{*}$ & 0,67 \\
& Pastejo & $19,6 \pm 16,9$ & $39,00^{* *}$ & $-0,369^{*}$ & 0,64 \\
Alta & Controle & $226,5 \pm 101,7$ & $110,50^{*}$ & $2,210^{*}$ & 0,64 \\
& Pastejo & $11,6 \pm 9,7$ & $24,67^{* * *}$ & $-0,248^{* * *}$ & 0,89 \\
Muito alta & Controle & $774,5 \pm 252,1$ & $476,50^{* *}$ & $5,676^{*}$ & 0,68 \\
& Pastejo & $221,0 \pm 85,0$ & $174,75^{*}$ & $0,881 \mathrm{NS}$ & 0,15
\end{tabular}

NS = Não significativo; ${ }^{*} \mathrm{P}<0,05 ;{ }^{* *} \mathrm{P}<0,01 ;{ }^{* * *} \mathrm{P}<0,001$.

3). No que diz respeito à identificação das fenofases reprodutivas as distribuições foram distintas nas infestações Baixa e Média (Fig. 3). Em contraste foi possível encontrar diferenças de vigor entre os tratamentos nos locais com as
Quadro 3. Valores e significância pelo teste de Wilcoxon (W) na comparação das variáveis qualitativas nos diferentes níveis de infestação entre as áreas de controle e pastoreio durante o inverno e primavera de 2010 (Experimento 2)

\begin{tabular}{lccc}
\hline Infestação & Fenofases reprodutivas & Vigor & Consumo \\
\hline Baixa & $60^{* *}$ & $42,5 \mathrm{NS}$ & $4^{* *}$ \\
Média & $60^{* * *}$ & $58^{* *}$ & $16^{*}$ \\
Alta & $36 \mathrm{NS}$ & $63^{* * *}$ & $28 \mathrm{NS}$ \\
Muito Alta & $41 \mathrm{NS}$ & $51^{*}$ & $8^{* *}$
\end{tabular}

NS= Não significativo; ${ }^{*} \mathrm{P}<0,05 ;{ }^{* *} \mathrm{P}<0,01 ;{ }^{* * *} \mathrm{P}<0,001$.

infestações mais altas por Senecio (Fig.4). Já no que se refere à avaliação de consumo no tratamento sob pastejo em comparação com o módulo mantido sem animais, chama a atenção um padrão peculiar de consumo nos primeiros quinze dias na infestação Alta (Fig.5).

\section{DISCUSSÃO}

Os experimentos foram conduzidos durante o inverno e a primavera porque são períodos reconhecidamente críticos
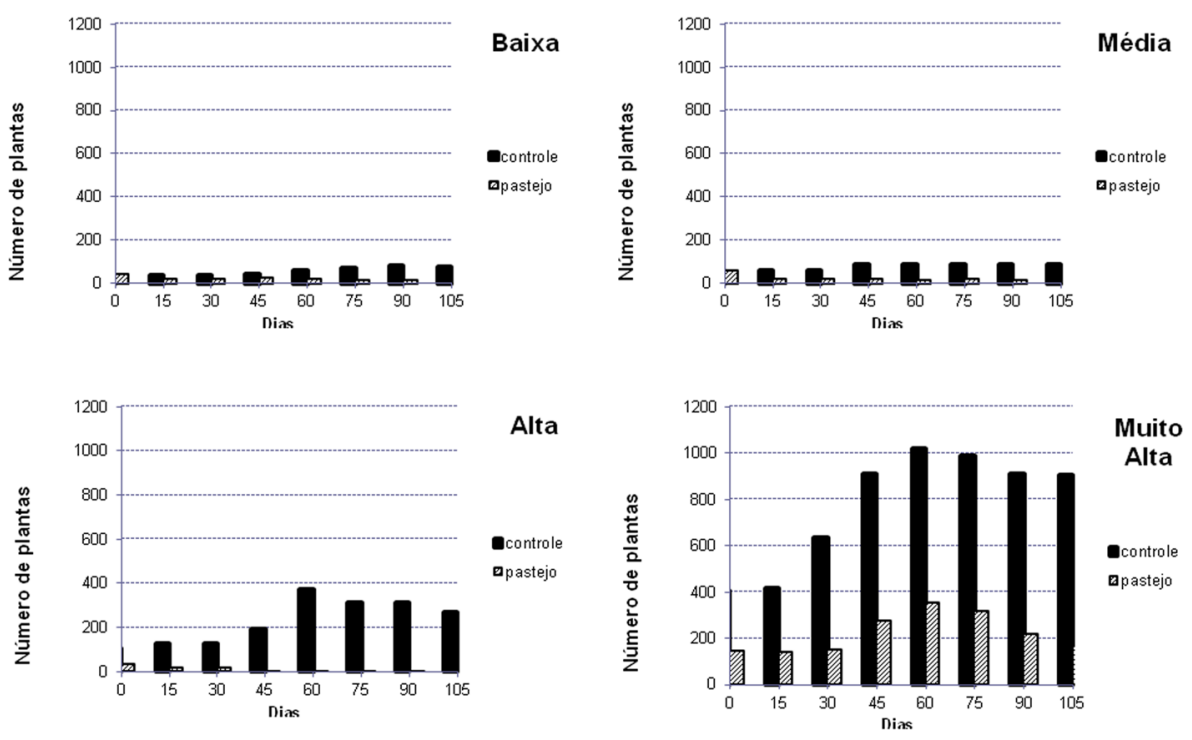

Fig.2. Variação do número de plantas de Senecio spp. nos módulos de Infestação Baixa, Média, Alta e Muito Alta submetidos ao pastejo ovino durante o inverno e primavera de 2010 e respectivos Controles (Experimento 2). são de sementes=2) em Senecio spp. nos módulos de Infestação Baixa, Média, Alta e Muito Alta submetidos ao pastejo ovino durante o inverno e primavera de 2010 e respectivos Controles (Experimento 2).

Fig.3. Variação das diferentes classes de fenofases reprodutivas (não ocorrência $=0$; botões florais, flores e frutos verdes $=1$; frutos maduros e disper-
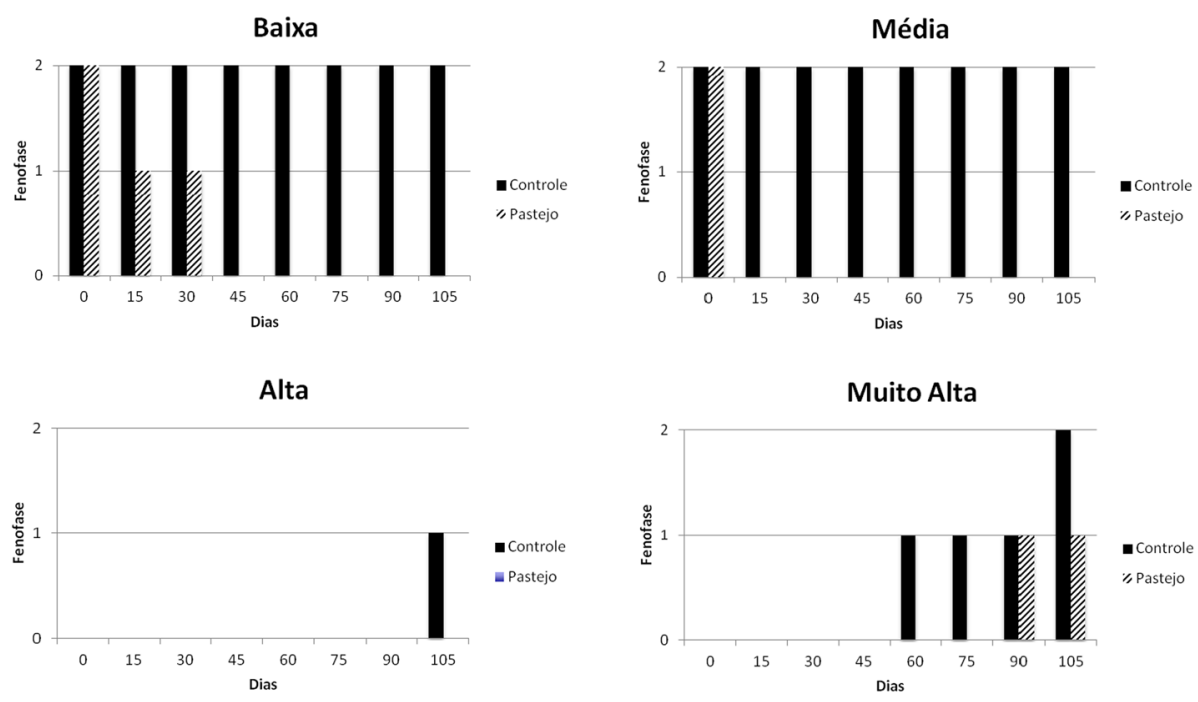
Fig.4. Variação das diferentes classes de vigor (fraco $=1$, moderado $=2$ e forte $=3$ ) em Senecio spp. nos módulos de Infestação Baixa, Média, Alta e Muito Alta submetidos ao pastejo ovino durante o inverno e primavera de 2010 e respectivos Controles (Experimento 2).

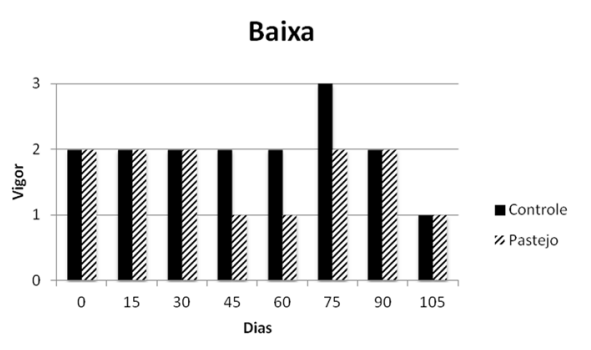

Alta

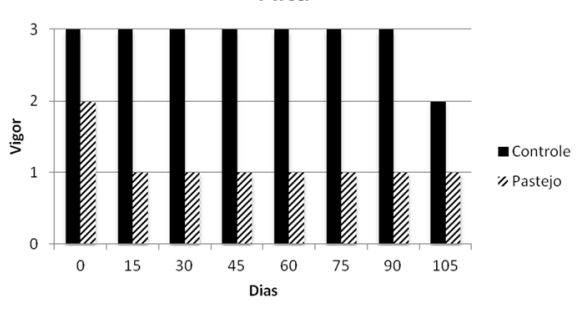

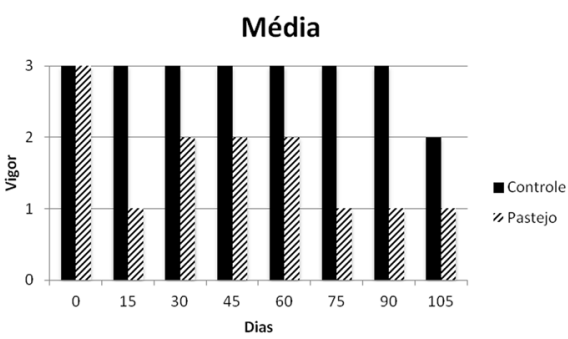



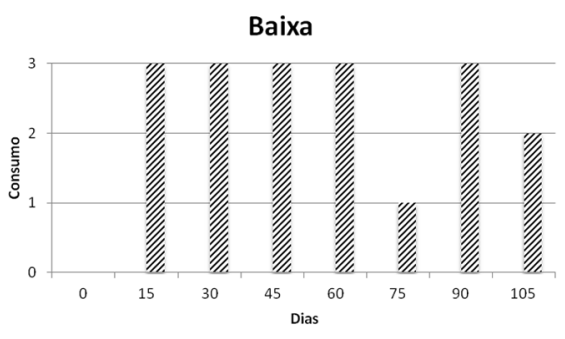

Alta

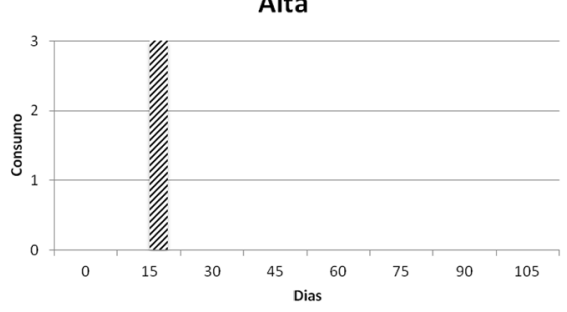

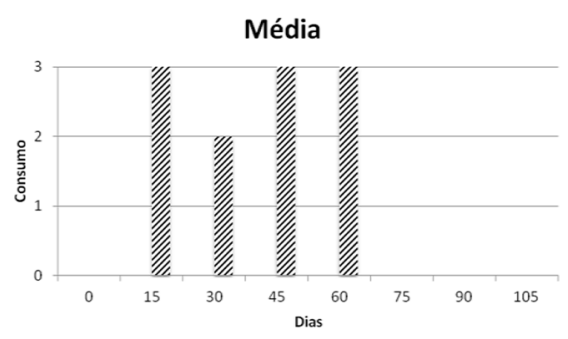

Muito Alta

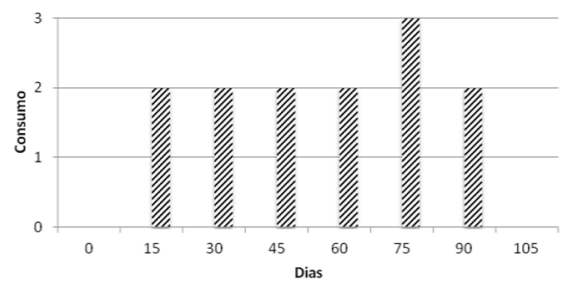

Fig.5. Variação das diferentes classes de consumo (não ocorrência $=0$, apenas ponteiros das plantas $=1$, parcial de ponteiros e partes aéreas $=2$, total das partes aéreas=3) em Senecio spp. nos módulos de Infestação Baixa, Média, Alta e Muito Alta submetidos ao pastejo ovino durante o inverno e primavera de 2010 (Experimento 2). para a ingestão e para a fenologia da planta, um pela falta de oferta adequada de pasto e pela maior emergência e potencialidade tóxica de Senecio spp., outro pela identificação facilitada durante a floração e quando pode-se melhor avaliar o comportamento de suas fases reprodutivas, respectivamente. A lotação experimental de dois ovinos em meio hectare, ao extrapolar para o manejo de uma propriedade, quatro ovinos/hectare, está dentro da lotação tradicionalmente usada nas propriedades criadoras de ovinos nessa região.

No primeiro ano de observação, primavera de 2009 (Fig.1), observa-se o nítido declínio do número de plantas após a introdução dos ovinos, embora se considere que naturalmente ocorre um declínio de Senecio spp. ao longo da primavera, após sua floração. Mesmo assim, os ovinos a consomem com intensidade semelhante em todos os níveis de infestação, apenas não havendo variação significativa na inclinação desse consumo no nível de infestação Baixa (Quadro 1), muito provavelmente pelo próprio parâmetro de infestação ser baixo.

No segundo ano experimental (inverno-primavera de 2010), quando novamente as áreas dos módulos foram fe- chadas, notou-se que todos os exemplares de Senecio spp. presentes, fossem plântulas ou plantas, não haviam sido consumidos ou perturbados, seis meses após interrupção do pastoreio ovino. Comparando-se o início e o final do experimento, observou-se o número de exemplares de Senecio spp. crescente nos controles e decrescente nos módulos com ovinos, demonstrando a interferência desse pastoreio nas parcelas experimentais (Fig.2). Ao compararmos a população da planta entre o inverno (0-60 dias) e a primavera (75-105 dias), depreende-se que o pastoreio já no inverno manteve ou diminuiu o número de exemplares de Senecio spp. até a primavera, enquanto no controle, onde não havia ovinos, houve um aumento expressivo da população de Senecio, portanto muito mais plantas podendo produzir flores e sementes no ambiente não pastoreado por ovinos.

Em relação às fenofases reprodutivas, foi baixa sua expressão nas infestações alta e muito alta durante o início do experimento (inverno), resultando em distribuições semelhantes nos dois tratamentos (Quadro 3). Isto, possivelmente, deve-se ao fato desses módulos experimentais terem sido constituídos exclusivamente por S. oxyphyllus e na fase inicial de plântula, cujo período de reprodução, em 
condições normais, é restrito ao mês de outubro, primavera (Matzenbacher 1998). A espécie S. madagascariensis, dominante nas infestações baixa e média, tem capacidade reprodutiva durante todo o ano (Villalba \& Fernández 2005) e já manifestavam essas fases no início do experimento. Com o pastoreio iniciado no inverno, houve interferência negativa no desenvolvimento das plantas, que não atingiram o estádio de floração após a introdução dos ovinos e as fenofases reprodutivas ocorreram de forma pouco expressiva na primavera e apenas em $S$. oxyphyllus na infestação muito alta, ao contrário de seus controles (Fig.3).

0 pastoreio desde o inverno permitiu que apenas algumas plantas chegassem viáveis à primavera nas infestações baixa e média. Na infestação alta, apesar do consumo não significativo (Quadro 3), essa população foi praticamente consumida quando da primeira leitura após a introdução dos ovinos, o que em parte se deve aos poucos exemplares na ocasião. $\mathrm{Na}$ infestação muito alta, embora com população reduzida em relação ao início do experimento no inverno, ainda havia muitos exemplares de Senecio e eram consumidos pelos ovinos apesar da boa oferta de pasto na primavera (Fig.5). Essa população ainda permanecia porque, provavelmente, o número de ovinos utilizados não tenha sido suficiente para o controle desde o inverno, o que explica a inclinação não significativa entre o consumo e o controle (Quadro 2), devendo-se testar um número maior de ovinos para esse nível de infestação.

0 pastoreio dos ovinos implica, também, no vigor das plantas (Fig.4). Aparentemente, enquanto as plantas não completam seu ciclo, o vigor mostra-se mais forte nas plantas não perturbadas no controle e mais fraco nas pastejadas no módulo com os ovinos, decaindo naturalmente nos controles após completarem seu ciclo vegetativo, já na primavera. Na infestação baixa, especialmente, apesar das diferenças entre o final do inverno e início da primavera, o vigor não foi significativo comparando-se a população do módulo com ovinos e o controle (Quadro 3), muito provavelmente pelo pequeno número de exemplares nesse nível de infestação. Nas observações de campo, em relação ao tratamento aplicado, notou-se o vigor mais forte nas plantas do controle e, nos módulos, antes da introdução dos ovinos. Mesmo após o início desse pastoreio, porém, normalmente as plântulas não foram perturbadas e se mantiveram vigorosas, especialmente as que se localizavam em áreas classificadas como protegidas, uma vez que continham plantas espinhosas e bem desenvolvidas, condição de pastagem não preferencial para ovinos. A classificação do vigor decaía a partir do desenvolvimento e passagem do estádio de plântula a planta jovem, quando se sobressaía na pastagem e eram mais frequentemente perturbadas pelo pastoreio. Contudo, mesmo as plantas consumidas que produziram brotos vegetativos e originaram novos ramos, mas expostas ao pastoreio ovino contínuo, não apresentaram bom vigor e a maioria não se estabeleceu no ambiente. Além disso, observou-se que os ovinos consumiam exemplares de Senecio que não estavam nos pontos fenológicos, incluindo $S$. brasiliensis e $S$. selloi, cujas espécies sofreram, também, influência negativa no seu desenvolvimento e tiveram as fenofases reprodutivas inibidas. 0 fato de ovinos não pastorearem, em condições habituais, pastagens altas, grossas e espinhentas, supõe que o pastoreio conjunto com bovinos ou associado a roçadas, mantenha a área adequada ao pastejo ovino, com exemplares jovens de Senecio spp. acessíveis ao seu controle. Essas plantas têm capacidade de propagação vegetativa (Silva \& Silva 2009) e o uso de roçadas associadas ao pastoreio ovino é importante, estrategicamente antes da floração, uma vez que evitam a produção e dispersão de sementes.

Na propriedade A dos experimentos, S. madagascariensis parece ser dominante entre as espécies de Senecio existentes, ao passo que cerca de 10 anos atrás, essa espécie não existia na propriedade, o que salienta o caráter agressivo da planta como invasora (Villalba \& Fernández 2005, Mäder et al. 2011), além de tóxica, o que exige medidas urgentes para seu controle.

Nas espécies de Senecio estudadas no RS, as fenofases vegetativas de emergência e crescimento, de maior frequência no outono-inverno, são potencialmente mais tóxicas (Karam et al. 2004), exceção à $S$. madagascariensis, cujo conteúdo de alcaloides é maior nas flores, especialmente na primavera, assim como no conjunto das partes aéreas (Karam et al. 2011a). Se ovinos usados para o controle da planta a consumirem na fase reprodutiva, normalmente primavera para a maioria das espécies, serão potenciais dispersores da mesma, como foi observado com S. jacoba$e a$, cujas sementes não são danificadas no trato digestivo dos ovinos e podem germinar (Harper \& Wood 1957). No $\mathrm{RS}$, as fenofases reprodutivas de grande parte das espécies de Senecio se concentram de setembro a dezembro (Matzenbacher1998), portanto, é recomendável o pastoreio com ovinos antes dessa época, o que reduzirá a produção de sementes, valorizando essa prática como alternativa de controle de Senecio spp.

Quanto ao uso de ovinos como controladores, no entanto, deve ser considerada a possibilidade de que em áreas muito infestadas por Senecio spp. os animais possam intoxicar-se (Ilha et al. 2001, Allan et al. 2005, Méndez \& Riet-Correa 2008, Grecco et al. 2011) e, especificamente por $S$. madagascariensis, se pastorearem em área muito infestada por duas estações seguidas (Motooka et al. 1999). Isso exige maior atenção à possibilidade da intoxicação nessa espécie, o que não foi acompanhado nesses experimentos, mas, aparentemente, os animais utilizados não apresentaram sinais de seneciose durante os dois anos do estudo.

\section{CONCLUSÕES}

O pastoreio ovino interfere negativamente no desenvolvimento das espécies de Senecio acompanhadas e, no inverno, impede as futuras fenofases reprodutivas das plantas ou estas ocorrem de forma pouco vigorosa, com pouca produção de sementes, assim como o vigor decai especialmente a partir da passagem de plântula a planta jovem e se exposta ao pastoreio ovino contínuo, a maioria não se estabelece no ambiente.

Considerando as condições fenológicas, ambientais e de maior risco para os bovinos, o controle de Senecio spp. com ovinos, no inverno, é mais eficaz do que na primavera e se constitui numa alternativa de controle biológico que pode 
contribuir na redução da infestação a médio e longo prazo no RS e, consequentemente, o prejuízo à bovinocultura.

Agradecimentos.- Ao Condomínio Rural Rossell e Romero pela área experimental. Trabalho financiado pelo INCT/CNPq (Proc. no 573534/20080 e no 551824/2010-7).

\section{REFERÊNCIAS}

Allan H., Launders T. \& Walker K. 2005. Fireweed. Primefact 126:1-8. State of New South Wales. Disponível em <http://www.dpi.nsw.gov.au/> Acesso em: set. 2010

Basile J.R., Diniz J.M.F., Okano W., Cirio S.M. \& Leite L.C. 2005. Intoxicação por Senecio spp. (Compositae) em bovinos no sul do Brasil. Acta Scient. Vet. 33:57-62.

Boldrini I.I., Ferreira P.M.A., Andrade B.O., Schneider A.A., Setubal R.B., Trevisan R. \& Freitas E.M. 2010. Bioma Pampa: diversidade florística e fisionômica. Palotti, Porto Alegre. 64p.

Borgignon O.J. \& Piccolo A.L.G. 1981. Fenologia de Hydrocotyle leucocephala Cham. Rodriguésia 33(56):91-99.

Callegari-Jacques S.M. 2003. Bioestatística: princípios e aplicações. Artmed, Porto Alegre. 255p.

Cruz C.E.F., Karam F.C., Dalto A.C., Pavarini S.P., Bandarra P.M. \& Driemeier D. 2010. Fireweed (Senecio madagascariensis) poisoning in cattle. Pesq. Vet. Bras. 30(1):10-12.

Grecco F.B., Schild A.L., Estima-Silva P., Marcolongo-Pereira C., Soares M.P. \& Sallis E.S.V. 2010. Aspectos epidemiológicos e padrões de lesões hepáticas em 35 surtos de intoxicação por Senecio spp. em bovinos no sul do Rio Grande do Sul. Pesq. Vet. Bras. 30(5):389-397.

Grecco F.B., Estima-Silva P., Marcolongo-Pereira C., Soares M.P., Collares G. \& Schild A.L. 2011. Seneciose crônica em ovinos no sul do Rio Grande do Sul. Pesq. Vet. Bras. 31(4):326-330.

Harper J.L. \& Wood W.A. 1957. Biological flora of the British Isles: Senecio jacobaea L. J. Ecology 45:617-637.

Ilha M.R., Loretti A.P., Barros S.S. \& Barros C.L. 2001. Intoxicação espontânea por Senecio brasiliensis (Asteraceae) em ovinos no Rio Grande do Sul. Pesq. Vet. Bras. 21:123-138.

Karam F.S.C., Méndez M.C., Jarenkow J.A. \& Riet-Correa F. 2002. Fenologia de quatro espécies tóxicas de Senecio (Asteraceae) na região Sul do Rio Grande do Sul. Pesq. Vet. Bras. 22(1):33-39.

Karam F.S.C., Soares M.P., Haraguchi M., Riet-Correa F., Méndez M.C. \& Jarenkow J.A. 2004. Aspectos epidemiológicos da seneciose na região sul do Rio Grande do Sul. Pesq. Vet. Bras. 24(4):191-198.

Karam F.S.C. \& Motta A.C. 2011. Pyrrolizidine alkaloids poisoning in cattle in the state of Rio Grande do Sul, Brazil, p.175-178. In: Riet-Correa F., Pfister J., Schild A.L. \& Wierenga T. (Eds), Poisoning by Plants, Mycotoxins and Related Toxins. CAB International, Wallingford, UK.

Karam F.S.C., Haraguchi M. \& Gardner D. 2011a. Seasonal variation in pyrrolizidine alkaloid concentration and plant development in Senecio madagascariensis Poir. (Asteraceae) in Brazil, p.179-185. In: Riet-Correa F., Pfister J., Schild A.L. \& Wierenga T. (Eds), Poisoning by Plants, Mycotoxins and related Toxins. CAB International, Wallingford, UK.

Karam F.C., Schild A.L. \& Mello J.R.B. 2011b. Intoxicação por Senecio spp. em bovinos no Rio Grande do Sul: condições ambientais favoráveis e medidas de controle. Pesq. Vet. Bras. 31(7):603-609.

Lucena R.B., Pierezan F., Kommers G.D., Irigoyen L.F., Fighera R.A. \& Barros C.S.L. 2010a. Doenças de bovinos no Sul do Brasil: 6.706 casos. Pesq. Vet. Bras. 30(5):428-434.

Lucena R.B., Rissi D.R., Maia L.A., Flores M.M., Dantas A.F.M., Nobre V.M.T., Riet-Correa F. \& Barros C.S.L. 2010b. Intoxicação por alcalóides pirrolizidínicos em ruminantes e equinos no Brasil. Pesq. Vet. Bras. 30(5):447452.

Madanes N., Vicari R. \& Bonaventura S.M. 1996. Fenologia de las especies de los bordes de caminos en agroecosistemas y su relación con los parámetros climáticos. Parodiana 9(1/2):149-158.

Matzenbacher N.I. 1998. O complexo "Senecionoide" (Asteraceae-Senecioneae) no Rio Grande do Sul, Brasil. Tese de Doutorado em Botânica, Instituto de Biociências, UFRGS, Porto Alegre. 274p.

Matzenbacher N.I. \& Schneider A.A. 2008. Nota sobre a presença de uma espécie adventícia de Senecio (Asteraceae) no Rio Grande do Sul, Brasil. Revta Bras. Biociênc. 6:111-115.

Matzenbacher N.I., Lima L.F.P., Dettke G.A., Durigon J., Kieling-Rubio M.A. \& Trevisan R. 2011. Flórula da Fazenda São Maximiano, Guaíba, Rio Grande do Sul, Brasil. Ediurcamp, Bagé. 106p.

Mäder G., Castro L., Bonatto S.L. \& Freitas L.B. 2011. Origin and population structure of invasive fireweed (Senecio madagascariensis) in Brazil. 57응 Congresso Brasileiro de Genética, Águas de Lindóia, SP, Brasil, p.168. (Resumo)

Méndez M.C. \& Riet-Correa F. 2008. Plantas Tóxicas e Micotoxicoses. 2ª̣ ed. Editora e Gráfica Universitária, Pelotas. 298p.

Motooka P., Nagai G., Onuma K., DuPonte M., Kawabata A. \& Fukumoto G. 1999. Control of Fireweed (Senecio madagascariensis). Hawaii Department of Agriculture, and University of Hawaii at Manoa (CTAHR). Weed Control WC-2.

Mueller-Dombois D. \& Ellenberg H. 1974. Aims and methods of vegetation ecology. John Wiley, New York. 547p.

Pedroso P.M.O., Pescador C.A., Oliveira E.C., Sonne L., Bandarra P.M., Raymundo D.L. \& Driemeier D. 2007. Intoxicações naturais por plantas em ruminantes diagnosticadas no Setor de Patologia Veterinária. Acta Scient. Vet. 35(2):213-218.

R Development Core Team 2011. R: A language and environment for statistical computing. R Foundation for Statistical Computing, Vienna, Austria. ISBN3-900051-07-0, URL http://www.R-project.org/

Rissi D.R., Rech R.R., Pierezan F., Gabriel A.L., Trost M.E., Brum J.S., Kommers G.C. \& Barros C.S.L. 2007. Intoxicações por plantas e micotoxinas associadas a plantas em bovinos no Rio Grande do Sul: 461 casos. Pesq. Vet. Bras. 27:261-268.

Santos J.C.A., Riet-Correa F., Simões S.V.D. \& Barros C.S.L. 2008. Patogênese, sinais clínicos e patologia das doenças causadas por plantas hepatotóxicas em ruminantes e equinos no Brasil. Pesq. Vet. Bras. 28(1):1-14.

Silva A.A. \& Silva J.F. 2009. Tópicos em Manejo de Plantas Daninhas. Editora UFV, Viçosa. 367p.

Villalba J. \& Fernández G. 2005. Otra flor amarilla peligrosa: Senecio madagascariensis. Tambo 150:46-48. 